\title{
Prime Focus Spectrograph (PFS): the metrology camera system
}

\section{Wang, Shiang-Yu, Chou, Chueh-Yi, Chang, Yin-Chang, Huang, Pin-Jie, Karr, Jennifer, et al.}

Shiang-Yu Wang, Chueh-Yi Chou, Yin-Chang Chang, Pin-Jie Huang, Jennifer Karr, Chi-Hung Yan, James E. Gunn, Craig Loomis, Robert H. Lupton, Hassan Siddiqui, Shu-Fu Hsu, Yen-Shan Hu, Daniel J. Reiley, Naoyuki Tamura, Yuki Moritani, Naruhisa Takato, "Prime Focus Spectrograph (PFS): the metrology camera system," Proc. SPIE 11447, Ground-based and Airborne Instrumentation for Astronomy VIII, 1144784 (13 December 2020); doi: $10.1117 / 12.2561191$ 


\title{
Prime Focus Spectrograph (PFS): The Metrology Camera System
}

\author{
Shiang-Yu Wang*a, Chueh-Yi Chou ${ }^{\mathrm{a}}$, Yin-Chang Changa, Pin-Jie Huanga , Jennifer Karr ${ }^{\mathrm{a}}$, Chi-Hung \\ Yan $^{\mathrm{a}}$, James E. Gunn ${ }^{\mathrm{b}}$, Craig Loomis ${ }^{\mathrm{b}}$, Robert Lupton ${ }^{\mathrm{b}}$, Hassan Siddiqui ${ }^{\mathrm{b}}$ Shu-Fu Hsu' ${ }^{\mathrm{a}}$, Yen-Shan \\ $\mathrm{Hu}^{\mathrm{a}}$, Daniel J. Reiley ${ }^{\mathrm{c}}$ Naoyuki Tamura ${ }^{\mathrm{d}}$, Yuki Moritani ${ }^{\mathrm{d}}$, Naruhisa Takato ${ }^{\mathrm{e}}$ \\ ${ }^{a}$ Academia Sinica, Institute of Astronomy and Astrophysics, P. O. Box 23-141, Taipei, Taiwan; \\ 'Princeton University, Princeton, New Jersey, 08544, USA; \\ ${ }^{\mathrm{c} C a l i f o r n i a}$ Institute of Technology, 1200 E California Blvd, Pasadena, CA 91125, USA; \\ ${ }^{\mathrm{d}}$ Kavli Institute for the Physics and Mathematics of the Universe (WPI),The University of Tokyo \\ Institutes for Advanced Study, The University of Tokyo, Kashiwa, Chiba 277-8583, Japan; \\ eSubaru Telescope, National Astronomical Observatory of Japan, 650 North A'ohoku Place Hilo, HI \\ 96720, U.S.A.
}

\begin{abstract}
The Prime Focus Spectrograph (PFS) is a new optical/near-infrared multi-fiber spectrograph designed for the prime focus of the $8.2 \mathrm{~m}$ Subaru telescope. PFS will cover a 1.3 degree diameter field with 2394 fibers to complement the imaging capabilities of Hyper SuprimeCam. To retain high throughput, the final positioning accuracy between the fibers and observing targets of PFS is required to be less than $10 \mu \mathrm{m}$. The metrology camera system (MCS) serves as the optical encoder of the fiber positioners for configuring of fibers. The MCS locates at the Cassegrain focus of the Subaru telescope to cover the whole focal plane with one 50M pixel CMOS sensor. The information from MCS will be fed into the fiber positioner control system for closed loop control. The MCS was delivered to Subaru Observatory in Apr. 2018 and it had two engineering runs in Oct. 2018 and Aug. 2019. The $1^{\text {st }}$ engineering run concluded that the original mirror supports need to be improved to provide better image quality. The newly designed mirror supports were installed before the $2^{\text {nd }}$ engineering run. The $2^{\text {nd }}$ engineering run result shows that the MCS overall position accuracy is better than $4 \mu \mathrm{m}$ and the image processing time is less than 4 seconds. The MCS is ready for the system integration with other PFS components.
\end{abstract}

Keywords: Metrology, CMOS sensor, multi-fiber, spectrograph, Schmidt telescope

\section{INTRODUCTION}

The Prime Focus Spectrograph (PFS) is a new prime focus optical/near-infrared multi-fiber spectrograph for the $8.2 \mathrm{~m}$ Subaru telescope'. The PFS will cover a 1.3 degree diameter field with 2394 fibers to complement the imaging capabilities of Hyper SuprimeCam². To retain high throughput, the final positioning accuracy between fibers and observing targets of PFS is required to be less than $10 \mu \mathrm{m}$. The metrology camera system (MCS) serves as the optical encoder of the fiber motors for the configuring of fibers ${ }^{3}$. The MCS is designed to provide the fiber position information within $5 \mu \mathrm{m}$ error over the $45 \mathrm{~cm}$ focal plane. The information from the metrology camera will be fed into the fiber positioner control system for closed loop control.

The MCS will be located at the Cassegrain focus of the Subaru telescope to cover the whole prime focal plane with a 50 M pixel Canon CMOS sensor. To reduce the high spatial frequency distortion of the wide field corrector (WFC), the aperture size of the metrology camera is set to be $380 \mathrm{~mm}$ which is the largest affordable aperture at the Suabru Cassegrain focus ${ }^{4}$. A Schmidt telescope type optical design was adapted to provide uniform image quality across the field with reasonable sampling of the point spread function (PSF). An achromatic lens set was designed to prevent the possible chromatic shift of the fiber spots due to the change of the wavelength of backlight LEDs. The mechanical design based on Invar structure and carbon fiber tubes provides stable structure over the temperature range under operating conditions. The CMOS sensor can be read in $0.8 \mathrm{~s}$ to reduce the overhead for the fiber configuration ${ }^{4}$. The positions of all fibers can be

*sywang@asiaa.sinica.edu.tw; phone 8862 2366-5338; fax 8862 2367-7849; www.asiaa.sinica.edu.tw

obtained within $0.5 \mathrm{~s}$ after the image readout is finished. This enables the overall fiber configuration to be less than 2 minutes.

Ground-based and Airborne Instrumentation for Astronomy VIII, edited by Christopher J. Evans,

Julia J. Bryant, Kentaro Motohara, Proc. of SPIE Vol. 11447, 1144784 · C 2020

SPIE · CCC code: $0277-786 \mathrm{X} / 20 / \$ 21 \cdot$ doi: $10.1117 / 12.2561191$ 
The PFS collaboration is led by the Kavli Institute for the Physics and Mathematics of the Universe of the University of Tokyo with international partners consisting of Academia Sinica, Institute of Astronomy and Astrophysics in Taiwan, Caltech/Jet Propulsion Laboratory, Princeton University, Johns Hopkins University and the NorthEast Participation Group in USA, the Chinese PFS Participating Consortium in China, the Laboratoire d'Astrophysique de Marseille in France, the Max Planck Institute for Astrophysics and Max Planck Institute for Extraterrestrial Physics in Germany, the National Astronomical Observatory of Japan/Subaru Telescope, the Brazilian consortium where Universidade de de São Paulo/Laboratório Nacional de Astrofísica.

\section{METROLOGY CAMERA OVERIEW}

The MCS has an aperture size of $380 \mathrm{~mm}$ in order to minimize the surface irregularity on the WFC lens surface while retaining the maximum affordable size of the telescope Cassegrain flange ${ }^{4}$. The optical layout is presented in the Fig. 1 below. The MCS is basically a Schmidt type reflector with a magnification of 0.037 and f/2.2 design. The first optics is the correction lens set including a Schmidt lens and two achromatic lenses that is used to maintain the image quality and minimize the potential centroid errors when the fiber illumination LEDs have wavelength shift in several microns. A spherical mirror reflects light to the sensor and a plano-convex lens serves as a field flattener to provide an uniform PSF across the entire field. Fig. 1 shows the ZEMAX simulated as-built PSFs taken from the field center and the edge. The MCS can provide an uniform PSF across the entire field with the FWHM 10 $\mu \mathrm{m}$. As an optical encoder of PFS fibers, the MCS shall provide a positioning accuracy better than $5 \mu \mathrm{m}$ over the $45 \mathrm{~cm}$ prime focal plane. The potential error sources of MCS position accuracy include the PSF centroid error, the WFC lens surface error, the chromatic error and the dome seeing effect. Details about the MCS optics, mechanical design can be found in previous proceedings ${ }^{4,5}$.
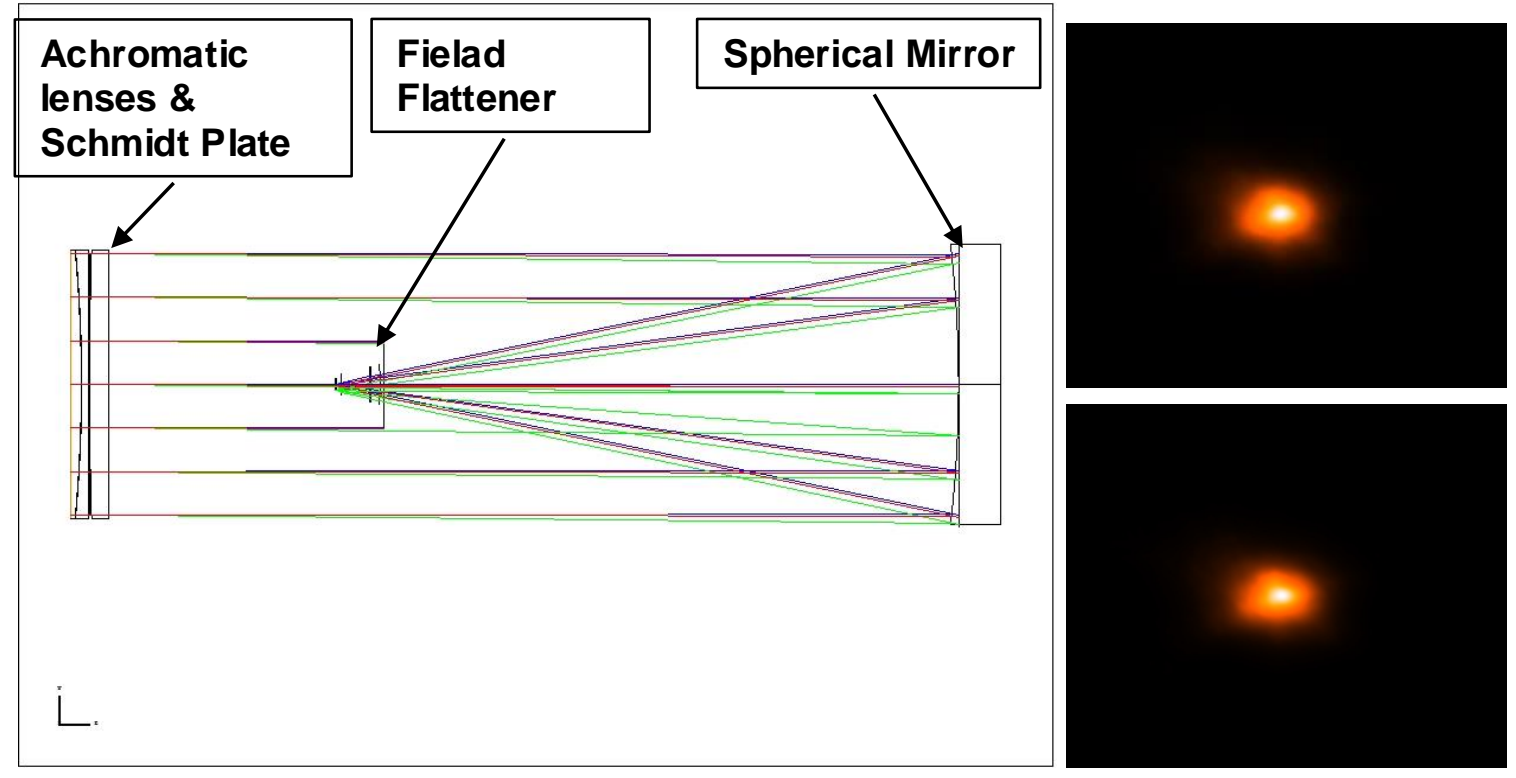

Figure 1. The optical layout of the MCS (left) and the simulated PFS at the field center (right top) and at the field edge (right bottom).

The MCS optics was delivered in 2016 and the system mechanical assembly was conducted in 2017. Fig. 2 shows the MCS mechanical layout, the 3D model and a picture with the MCS installed inside the telescope Cassegrain instrument box. 

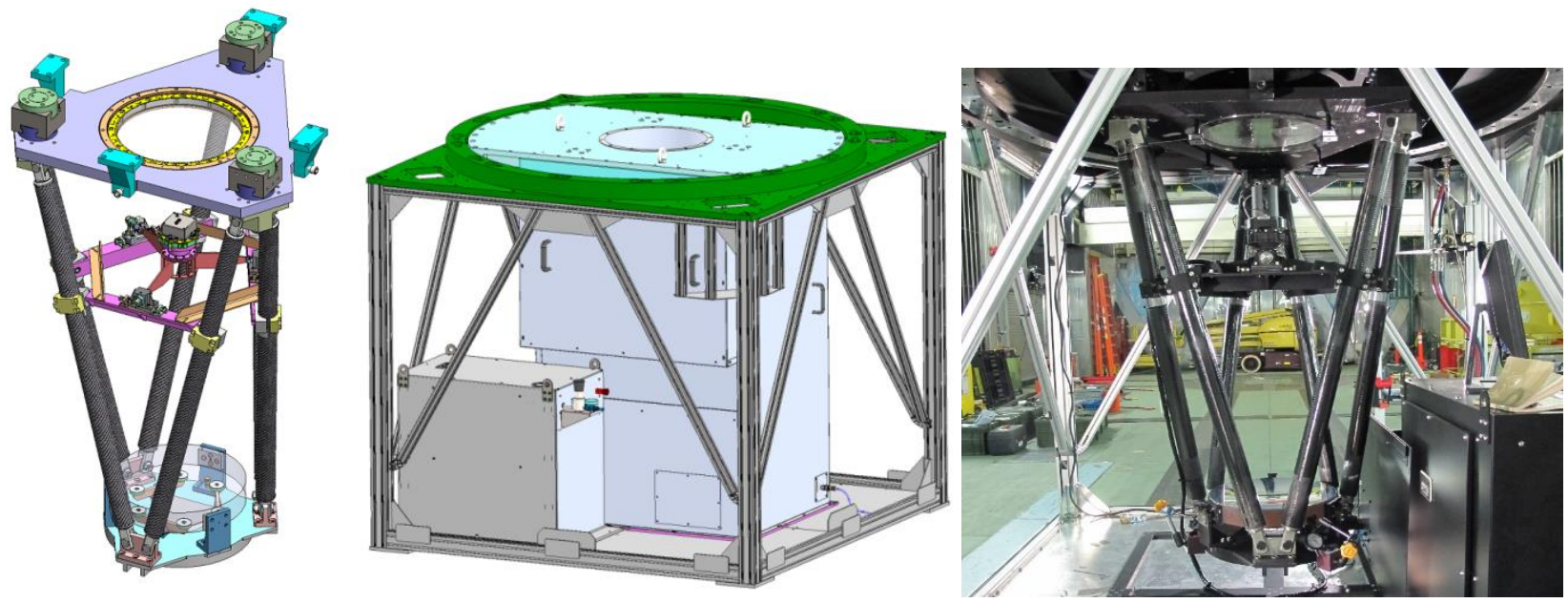

Fig. 2: The MCS mechanical layout (left). The 3D model of MCS inside the telescope Cassegrain instrument box (middle). The MCS installed inside the instrument box without the protection covers (right).

\section{ENGINEERING RUNS}

The MCS was assembled in Taiwan and passed the pre-ship review in March $2018^{6}$. After that, MCS was delivered to the observatory Hilo base in April, 2018. Due to the limited space in the Hilo base, we could only conduct basic alignment examination and no significant alignment issue was found. The MCS was then transported to the observatory for image quality verification. The alignment process went smoothly and the MCS performance fits the ZEMAX simulations. After the optical performance verification, the MCS is installed into the Cassegrain box as shown in Fig.3. The system was ready for the $1^{\text {st }}$ engineering run of MCS in October, 2018.

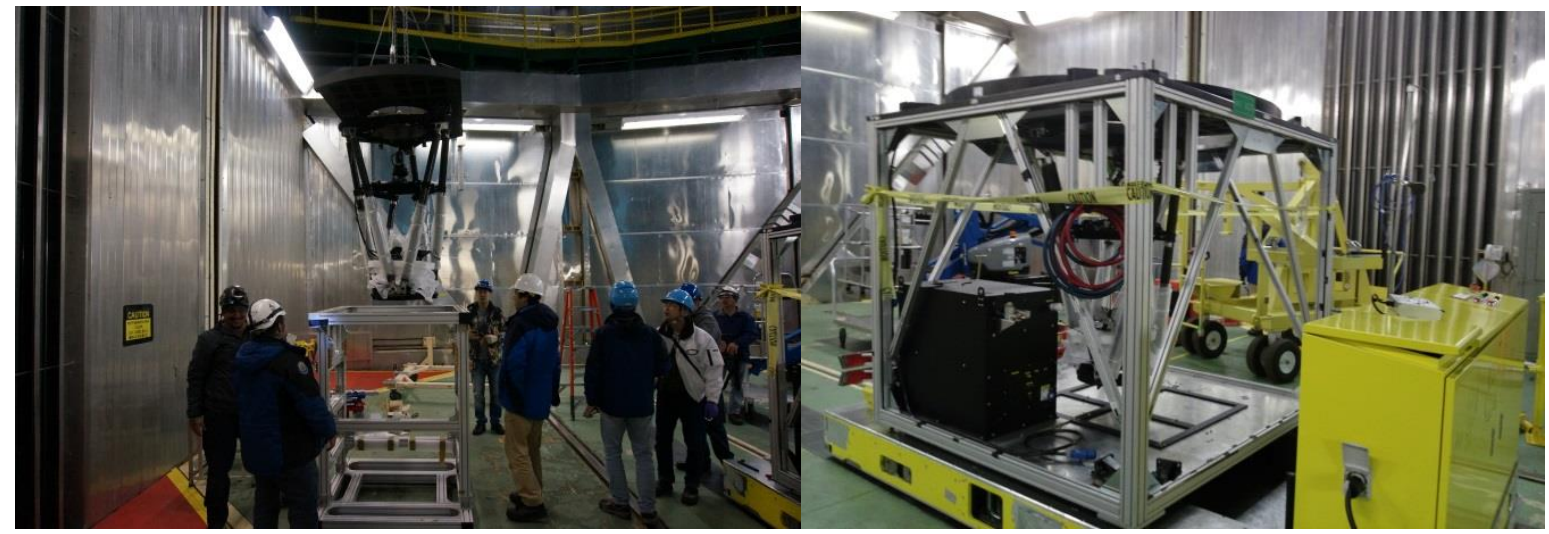

Fig. 3: The removal of MCS from the test frame (left). The MCS installed into the Subaru Cassegrain instrument box (right).

\subsection{The First Engineering Run}

Throughout the entire engineering run we adopted a backlit pinhole mask with 3576 holes placed at the telescope prime focal plane as the light source to simulate fiber spots. The size of the pinhole is the same as the actual PFS fiber and the space accuracy between the pinholes is better than $2 \mu \mathrm{m}$ such that this pinhole can be used for MCS calibrations. The ordinary spacing between the pinholes is $8 \mathrm{~mm}$, which is the same as the spacing between fibers at their home position. There are three regions with smaller spacing of $2 \mathrm{~mm}$, which is to simulate the minimum distance between two PFS fibers. The same mask was also used for the optical alignment before the engineering run. Fig. 4 left shows the layout of pinhole mask test assembly used for the engineering run. The green plate is the interface between the mask and the telescope. The distace between the interface plate to the pinhole mask is $234.06 \mathrm{~mm}$ which is the nominal distance for the PFS fibers. The 
telescope prime focal plane is $45 \mathrm{~cm}$ and the mask can not cover the it in a single position. Thus, the mask is positioned off the MCS center so that it can cover from the center to the edge and the full prime focal plane can be fully covered by rotating the mask. The configuration of the mask is shown in Fig. 4. The back light source of the mask has four different intensity settings to test the performance of centroiding algorithm with different signal to noise ratios. A CISCO switch and an IP power supply were used to remotely control the intensity of the back light.
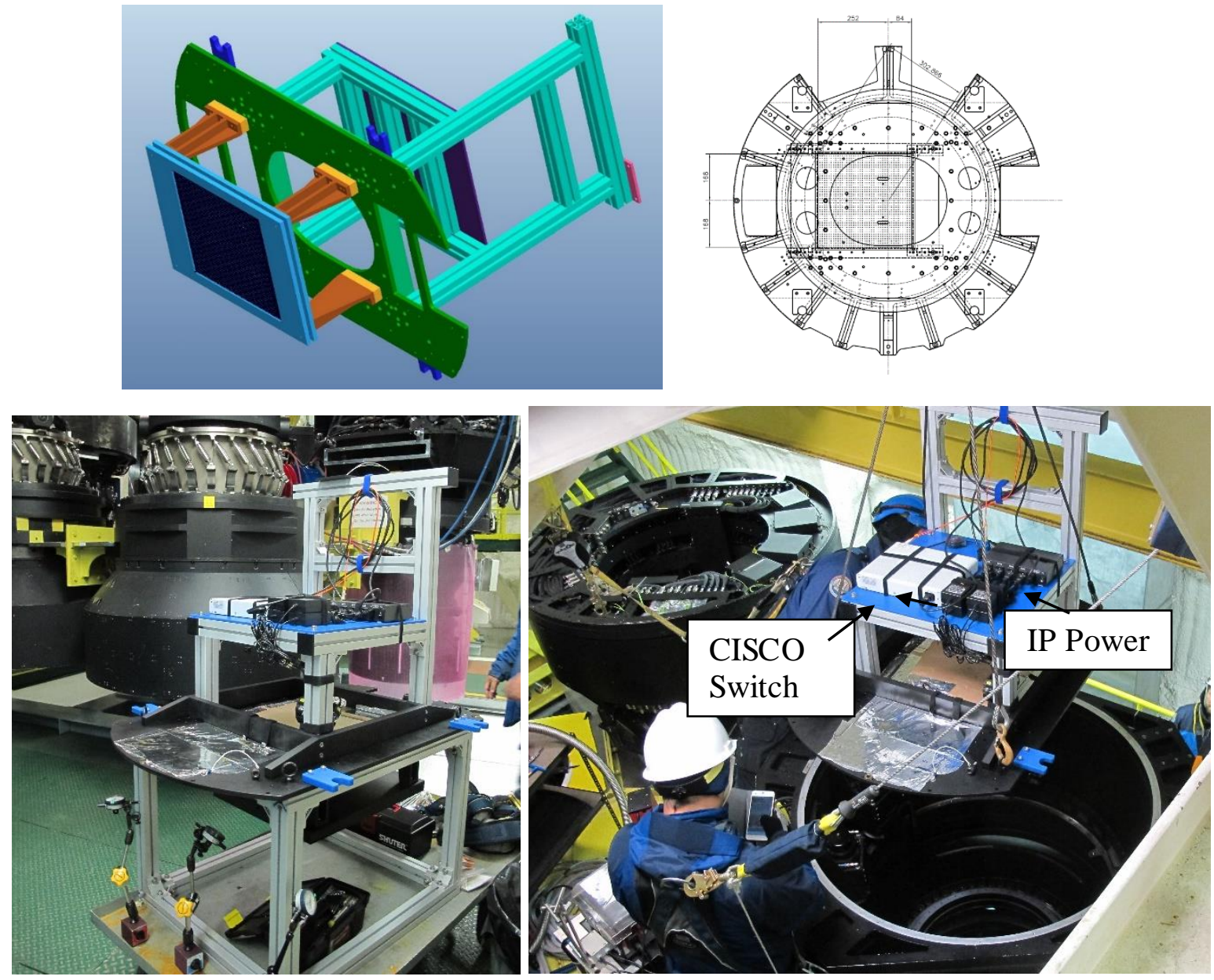

Fig. 4: Mechanical layout of the pinhole mask assembly used during the engineering run (top left). The pinehole mask is placed off to the opitcal axis to cover from the field center to the edge (top right). The actual pinhole mask supporting structure (bottom left). The mask structure installed into the POpt2 structure(bottom right).

It is also important to understand the deformation and shifts of the mask being installed on the supporting structure due to gravity under various elevation angles. Fig. 5 shows one example of the deformation analysis in $\mathrm{x}$ direction at elevation angle of 60 degrees. Most deformation happens along the $\mathrm{x}$ direction as the lateral shift and the $\mathrm{y}$ direction as the focus shift. The largest focal shift and lateral shift is around $300 \mu \mathrm{m}$ and $100 \mu \mathrm{m}$, respectively. The largest deformation of the mask itself is around $1 \mu \mathrm{m}$. The largest mask tilt generated by structure deformation is around 0.03 degrees. We fed these shifts and tilts into the ZEMAX simulation and discovered that the focus shift is negligible to the image quality change and the lateral shift is still within the tolerance range. The mask deformation is quite small and it will not affect the distortion map too much. The mask tilt will be the most significant error source for distrion map estimation. The 0.03 degrees tilt will yeild to $0.57 \mu \mathrm{m}$ change on MCS sensor or $15.5 \mu \mathrm{m}$ change on the prime focal plane at field edge. 


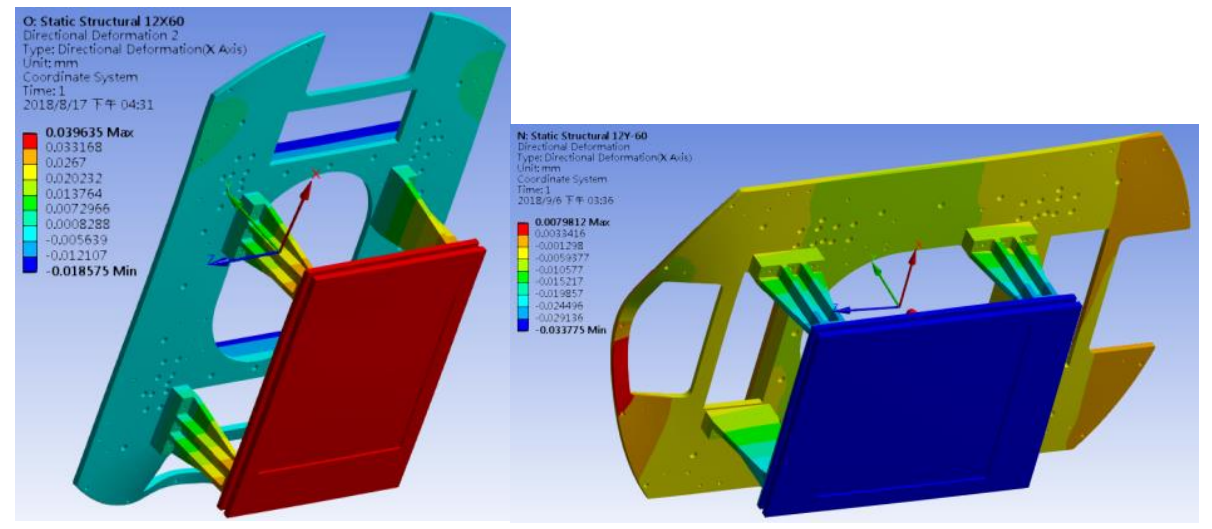

Fig. 5: One example of the mask assembly deformation at 60 degrees of elevation. For simplicity, only the deformation in $\mathrm{x}$ axis is presented. The analysis takes the deformation in three axis into consideration and fed the deformation back into ZEMAX simulation.

One example of the MCS image during the engineering run is shown in Fig. 6. The bottom corners are vignetted by the WFC. During the engineering run, we found that the image quality varies when the temperature and elevation angle changed. The PSF shape is also not spherical and shows several tail like structure as also shown in Fig. 6 . It is clear that the PFS also varied depending on the location of the field. The original centorid algotrithm which detects the PSF peak and perfrom serval 1-D Gaussian fitting along the PSF profile had difficulties in finding robust spot center in some corners of the image. Alternatively, we used the PSFEX (https://www.astromatic.net/software/psfex) to construct an averge PSF model for each single frame image and then re-do the centroid using the SExtractor (https://www.astromatic.net/software/sextractor). We were capable to obtained a more robust results, however, the image centroid acquisition time is more than 10 times longer than the required value. One example of the PSF FWHM distribution in $\mathrm{x}$ direction using the time consuming method is shown in the Fig. 6.
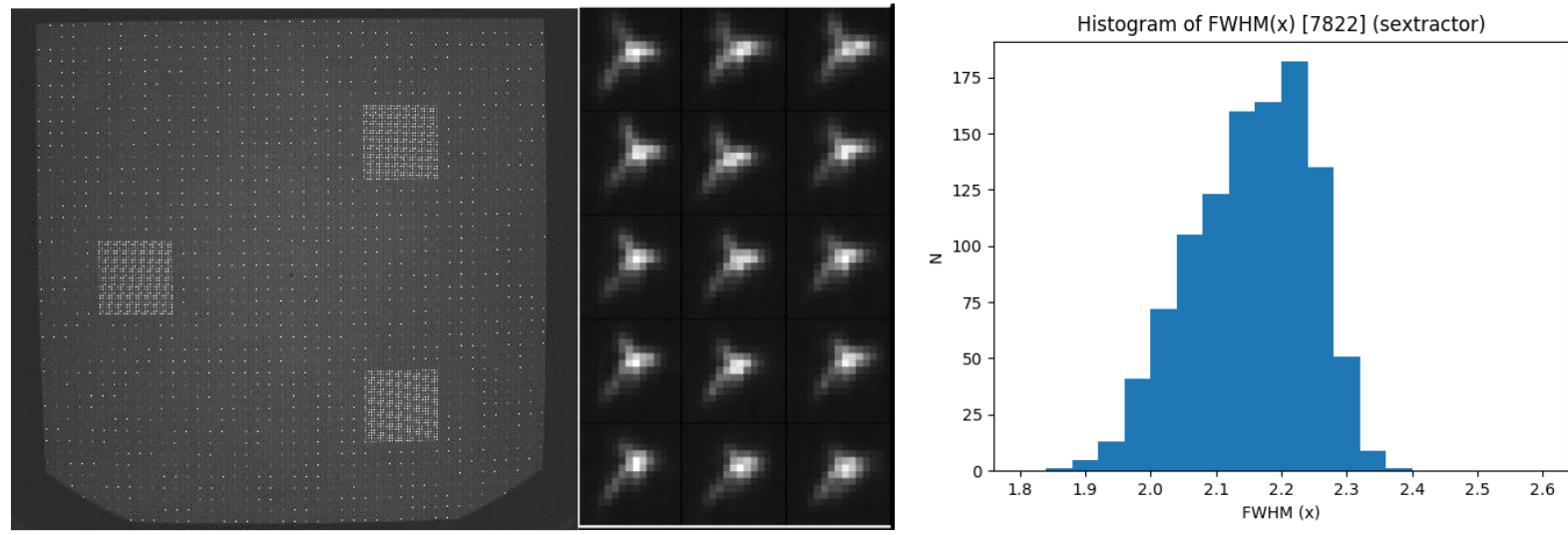

Fig. 6: Full view of the MCS image for the pinhole mask (left). Sample PSFs taken from the day 2 image of the engineering run (middle). Histogram of PSF FWHM distribution in $\mathrm{x}$ direction. The distribution peak locates at $\sim 2.2$ pixels.

After the image analysis, the PSF RMS spot motion with respect to observation time is presented in Fig. 7. Different symbols and colors indicate various elevation angles and expossure times, respectively. The vertical solid and dashed lines indicate the telescope dome and the air purge inside MCS protection cover conditions. Blue and red colors represent when the condition was on and off. From the data in Day 2, it is clear that the spot RMS motion decreases with the increase of exposure time as expected. On the other hand, the spot RMS motion changes significantly in Day 3 after the telescope dome was opened. After some inspections, this was associted with the PSF size variation. Other than that, the spot RMS motion in many cases can reach or below the required 0.02 pixel (green dashed line). The required performance is therefore 
achievable. We discovered the spot RMS motion has correlation in spot x-direction translation but no obvious correlation with the elevation angles as shown in the bottom panels in Fig.7. In addition, the RMS spot motion reduces when exposure time increases. These results indicate that the spot motion measured was genuinely dominated by the dome seeing effect and also the PFS shape. We concluded that the requirement of PSF centoid accuracy is acheivable, but the PSF shuld be more stable under various conditions. In that sense, the tail-like feature that significantly affects the centroid accuracy should be improved. After some analysis, we concluded this image degradation comes from the over constraint on the primary mirror by the mirror supports. We decided to design a new mirror support for replacement.
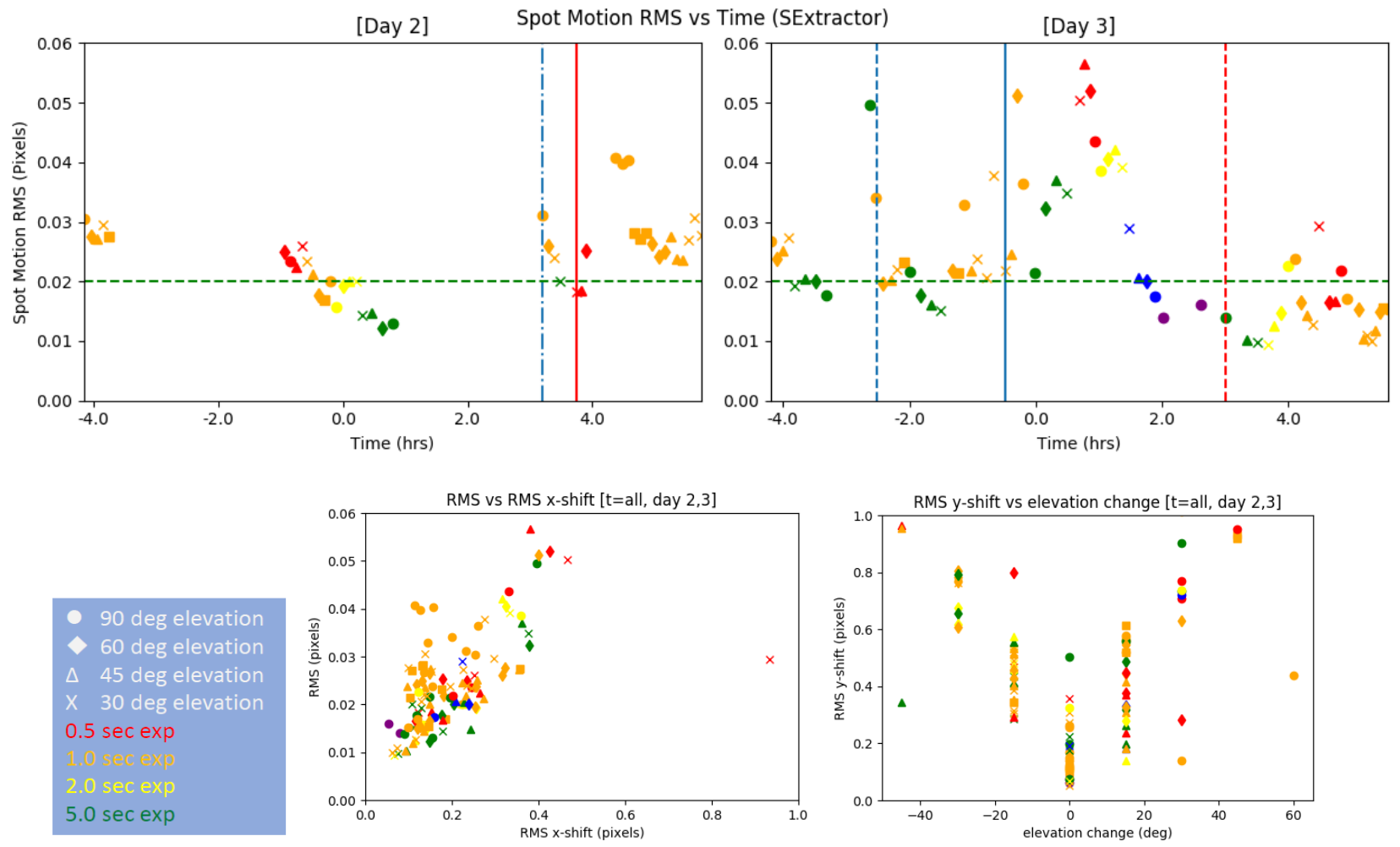

Fig. 7: PSF spot RMS motion with respect to time for Day 2 and 3 during the $1^{\text {st }}$ engineering run (top). The vertical solid and dashed lines indicate the telescope dome and the air purge conditions. Blue and red colors represent when the condition was on and off, respectively. Correlation of RMS with respect to spot $\mathrm{x}$-shift and telescope elevation angles. It is clear that the RMS spot motion has correlation with spot $\mathrm{x}$-motion and no significant correlation with telescope elevation angles. This indicates the spot RMS motion measured is likely due to the dome seeing effect.

\subsection{Mirror Support Replace ment \& Optical Alignment}

From the above section we have learned that the MCS primary mirror suffer too much stress from the original mirror support. In addition, the original mirror support adopts the traditional shimming mechanism that is time inefficient in adjusting mirror tilt/height. To solve these problems, a newly designed mirror support adopting a micrometer for mirror tilt/height adjustment and a ball head with a $\mathrm{V}$ groove support for the reduction of the constraint on the mirror was implemented. Fig. 8 shows the mechanical layout of the mirror support. A micrometer that controls the mirror support height with $10 \mu \mathrm{m}$ accuracy is used. This mechanism not only improves the mirror tilt/height adjustment accuracy but also makes the adjustment fast and repeatable. The ball head design (indicated by red circle) is supported by a V-shape groove and serves as a buffer between the mirror support and the mirror body. After the mirror support replacement, we conducted the optical alignment to retain the required image quality. 

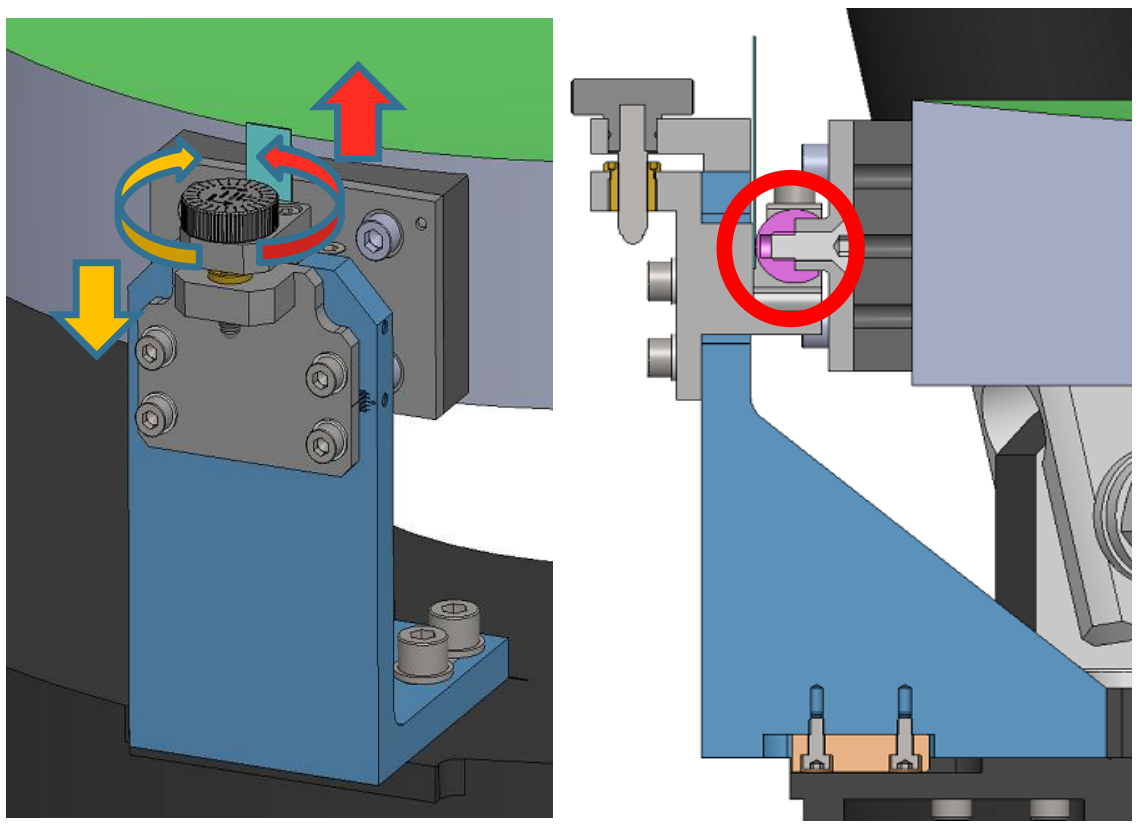

Fig. 8: The mechanical layout of the new mirror support (left). The micrometer can efficiently adjust the mirror tilt/height. Right panel: side view of the mirror support. The ball head design (indicated by red circle) is supported by a V-shape groove and serves as a buffer between the mirror support and the mirror body (right).

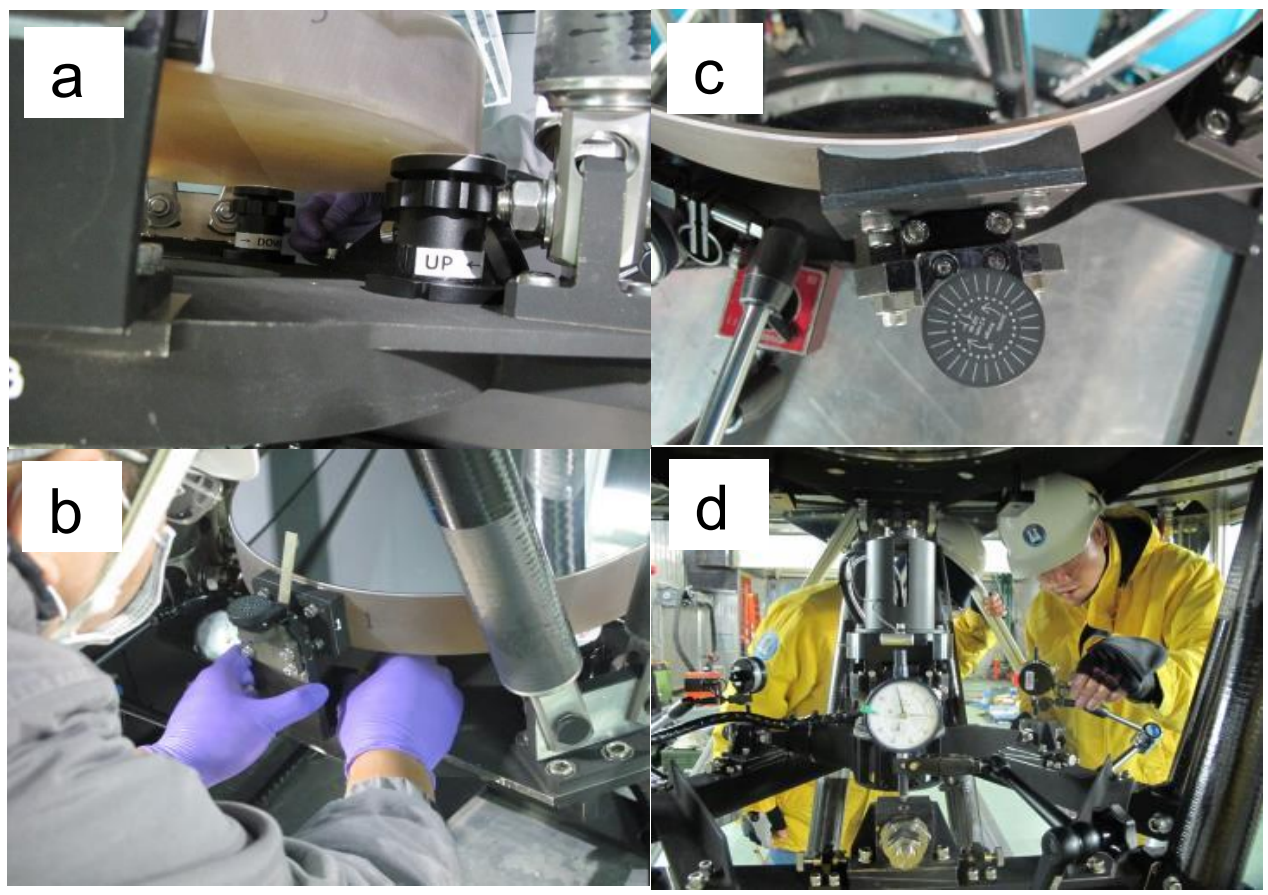

Fig. 9: pictures taken during different steps of the mirror support replacement and optical alignment procedure.

The alignment procedure was conducted smoothly. The micrometer makes the mirror height/tilt adjustment relatively easy and fast. We started the alignment from the mirror adjustment and we switched to the camera module assembly adjustment to fine tune the image quality and also the center position of MCS. Fig. 9 shows pictures taken at different steps during the 
mirror support replacement and the re-alignment procedure. After the adjustment and alignment, the MCS image center is $(-9,-16)$ pixels away from the telescope optical axis and well within the expected tolerances.

\subsection{Engineering Run 2 - Image Quality}

MCS had the $2^{\text {nd }}$ engineering run after the optical alignment process was completed in Aug. 2019. The test setup is the same as the engineering run 1. Figure 10 shows an example of the images taken during the $2^{\text {nd }}$ engineering run. The left panel shows the full view of the image which shows a magnification factor of 0.037 , consistent with the ZEMAX simulation. The middle left panel shows the PSFs of various locations of the pinhole mask image. The image quality is quite uniform across the field. The PSF is also quite stable under different elevation angles and temperatures. Although the PSF is not a perfect round shape, the centroid algorithm can deliver good accuracy without problems. The PSF FWHM distributions in $\mathrm{X}$ and $\mathrm{Y}$ direction are close to Gaussian with its peak at 2.3 pixels in $\mathrm{X}$ and 2.7 pixels in $\mathrm{Y}$. This shows the modification of the mirror support is effective and the data taken during the whole engineering run could be used for the MCS performance evaluation.
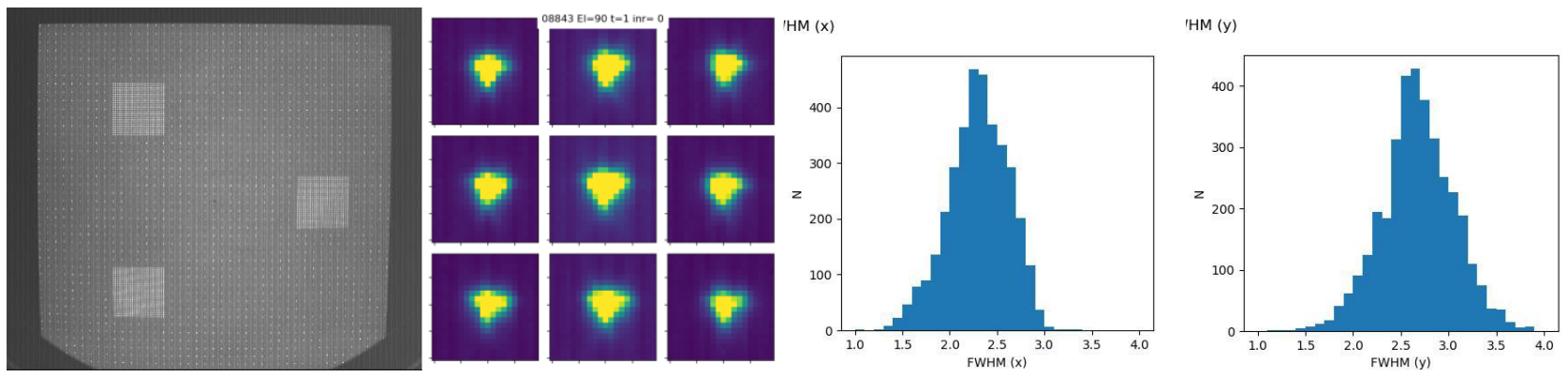

Fig. 10: A full image taken from $2^{\text {nd }}$ engineering run (left). The PSF samples with 1 s exposure from various corners and field center of the image (middle). The histograms of the PSF FWHM in $\mathrm{x}$ and y directions (right).

\subsection{Engineering Run 2 - Dome Seeing Measure ment \& Results}

One major task for the $2^{\text {nd }}$ engineering run is to measure the dome seeing effect, which is crucial to the MCS performance. During the $2^{\text {nd }}$ engineering run, the weather condition was good so that we were able to test MCS under different dome conditions. Like in the $1^{\text {st }}$ engineering run, the motion of the pinhole spots taken by the MCS system was used to investigate the dome seeing. The results for the three nights are presented in Fig. 11. Different shapes and colors of the symbols indicate various telescope elevation angles and exposure times. The green horizontal dashed line indicates the allocated error budget for the pure centroid accuracy; while the blue horizontal dashed line indicates the overall MCS centroiding requirement. The vertical blue dashed line shows when the telescope dome was open and the vertical solid line means we closed the dome. The green vertical dashed and solid lines indicate when the air purge inside MCS cage is on or off.

After the data analysis, we obtained several conclusions. In general, the dome seeing becomes better as night went on. The best seeing tends to happen by the end of the night. The dome seeing decreases when exposure time increases, and it seems to settle down when the exposure time is longer than $2 \mathrm{~s}$. The seeing dependence to the elevation angle is relatively low. We also noticed that the seeing settled in a relatively short time scale ( 10mins) after the telescope dome opened. In most cases, 1s exposure time can deliver dome seeing within the required MCS accuracy value of 0.04 pixels (blue dashed line). The centroid error (based on the smallest image movement obtained) is less than 0.01 pixels. The conclusion is that with $1 \mathrm{sec}$ exposures, the MCS can deliver the required accuracy under different observation conditions. 

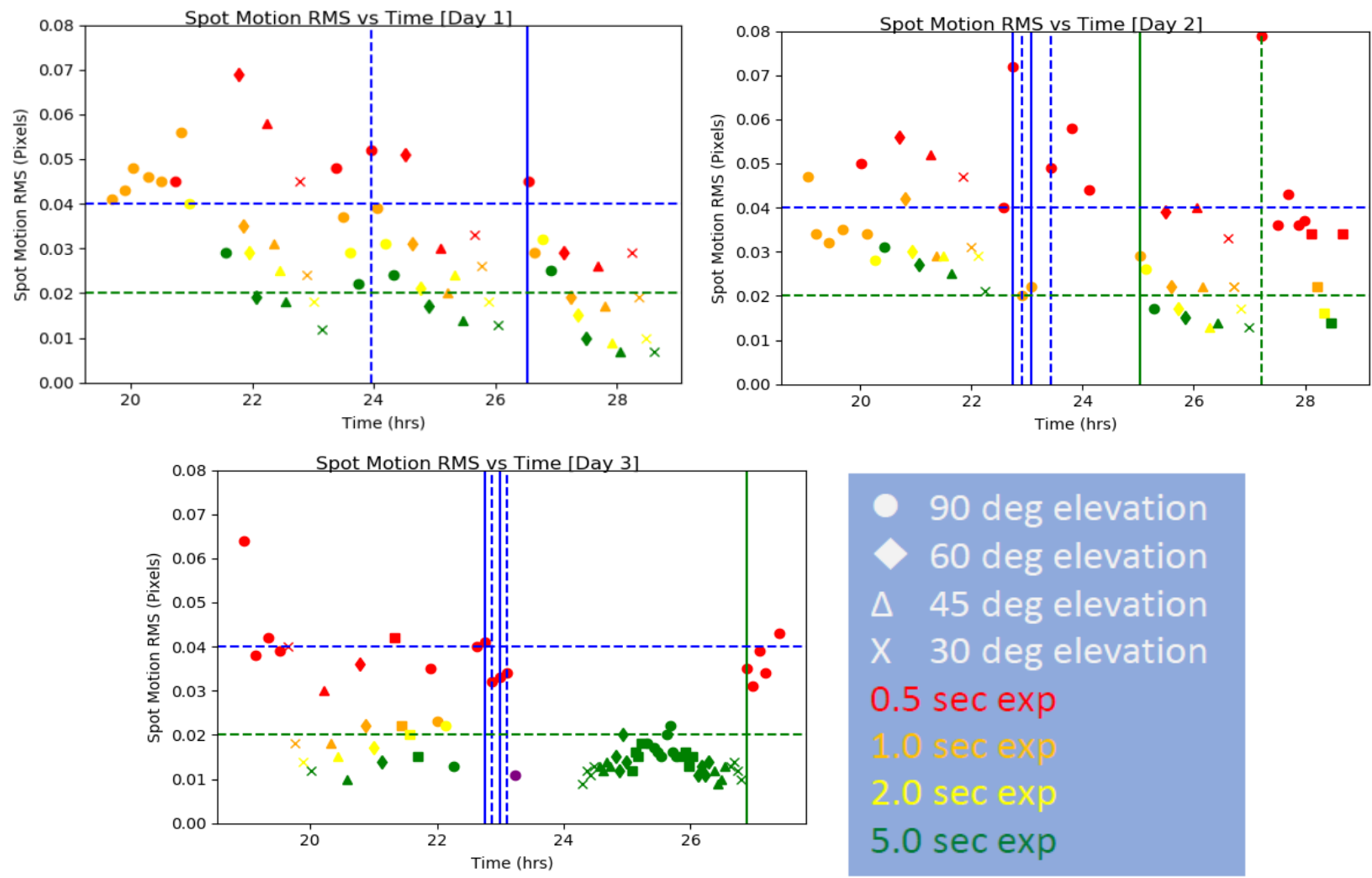

Fig. 11: The RMS spot motion in the unit of pixels versus time for the three days of the $2^{\text {nd }}$ engineering run. Different symbols and colors indicate various telescope elevation angles and exposure times.

\subsection{Image Processing Time}

The image readout time for $1 \mathrm{~s}$ exposure takes $3.36 \mathrm{~s}$ due to rolling shutter operation of the CMOS camera. It takes three frames to be saved and co-added. The centroid calculation time using a 16 core computer is $0.35 \mathrm{~s}$. It also takes $0.2 \mathrm{~s}$ for database insertion. This process needs to insert all the fiber positions and measured parameters into the database. Therefore, the total image processing time needed is $3.36+\mathbf{0 . 3 5}+\mathbf{0 . 2}=\mathbf{3 . 9 1}$ sec.

\section{SUMMARY}

The test result of MCS engineering runs were presented. The new mirror supports with a micrometer and ball-head structure was installed before the $2^{\text {nd }}$ engineering run to mitigate the image quality issue discovered during the $1^{\text {st }}$ engineering run. During the $2^{\text {nd }}$ engineering run, MCS can deliver stable images under various elevation angles and temperatures. The test under different conditions shows the MCS positioning accuracy is better than $4 \mu \mathrm{m}$ on the PFI focal plane, and the image process time is less than 4 seconds. In addition, the MCS center is aligned with prime focus ins trument rotator center within the expected tolerance. Based on the results shown above we conclude that MCS satisfies the expected requirements and is ready for the PFS operation.

\section{ACKNOWLEDGEMENT}

We gratefully acknowledge support from the Funding Program for World-Leading Innovative R\&D on Science and Technology(FIRST) "Subaru Measurements of Images and Redshifts (SuMIRe)", CSTP, Japan for PFS project. The work in ASIAA, Taiwan is supported by the Academia Sinica of Taiwan. 


\section{REFERENCES}

[1] Komi yama, Y., Aihara, H., Fujimori, H., Furusawa, H., Kamata, Y., Karoji, H., Kawanomoto, S., Mineo, S., Miyatake, H., Miyazaki, S., Morokuma, T., Nakaya, H., Nariai, K., Obuchi, Y., Okura, Y., Tanaka, Y., Uchida, T., Uraguchi, F., Utsumi, Y., Endo, M., Ezaki, Y., Matsuda, T., Miwa, Y., Yokota, H., Wang, S.-Y., Liaw, E. J., Chen, H.-Y., Chiu, C.-F., and Jeng, D.-Z., "Hyper Suprime-Cam: Camera Design," Proc. SPIE 7735, 77353F-1 (2010).

[2] Sugai, H. et al. "Prime focus spectrograph: Subaru's future," Proc. SPIE 8446, 84460Y (2012).

[3] Wang, S.-Y., Hu, Y.-S., Yan, C.-H., Chang, Y.-C. Tamura, N., Takato, N., Shimono, A., Karr, J., Ohyama, Y., Chen, H.-Y., Ling. H.-H., Karoji, H., Sugai, H., Ueda, A., "The metrology cameras for Subaru PFS and FMOS," Proc. SPIE 8446, 84464Z (2012).

[4] Wang, S.-Y., Chou, C.-Y., Chang, Y.-C., Huang, P.-J., Hu, Y.-S., Chen, H.-Y., Tamura, N., Takato, N., Ling, H.-H., Gunn, J. E., Karr, J., Yan, C.-H., Mao, P., Ohyama, Y., Karoji, H., Sugai, H., Shimono, A., "Metrology camera system of prime focus spectrograph for Subaru telescope," Proc. SPIE 9147, 91475S (2014).

[5] Wang, S.-Y., Chou, C.-Y., Huang, P.-J., Ling, H.-H., Karr, J., Chang, Y.-C., Hu, Y.-S., Hsu, S.-F., Chen, H.-Y., Gunn, J. E., Railey, D. J., Tamura, N., Takato, N., Shimono, A., " Metrology Camera System of Prime Focus Spectrograph for Subaru Telescope," Proc. SPIE SPIE 9908, 990881 (2016).

[6] Wang, Shiang-Yu; Chou, Richard C. -Y., Huang, P.-J., Chang, Y. -C., Ling, H. -H., Yan, C.-H., Karr, Jennifer, Hsu, S. -F., Chen, H. -Y., Hu, Y. -S., Gunn, J. E., Reiley, D. J., Tamura, N., Takato, N., Moritani, Y., Shimono, A., " Metrology Camera System of Prime Focus Spectrograph for Subaru Telescope," Proc. SPIE SPIE 10702, 107027H (2018). 